\title{
Rotational Spectroscopy of PAHs: Acenaphthene, Acenaphthylene and Fluorene
}

\author{
S. Thorwirth $\dagger$, P. Theuléł, C. A. Gottlieb, \\ M. C. McCarthy, and P. Thaddeus \\ Department of Engineering and Applied Sciences, Harvard University, \\ Pierce Hall, 29 Oxford Street, Cambridge, MA 02138, U.S.A. \\ Harvard-Smithsonian Center for Astrophysics, \\ 60 Garden Street, Cambridge, MA 02138, U.S.A. \\ email: sthorwirth@mpifr-bonn.mpg.de
}

\begin{abstract}
Pure rotational spectra of three polycyclic aromatic hydrocarbons - acenaphthene, acenaphthylene and fluorene - have been obtained by Fourier transform microwave spectroscopy of a molecular beam and subsequently by millimeter wave absorption spectroscopy for acenaphthene and fluorene. The data presented here will be useful for deep radio astronomical searches for PAHs employing large radio telecopes.
\end{abstract}

Keywords. astrochemistry — ISM: molecules — molecular data

Polycyclic aromatic hydrocarbons (PAHs) have been studied extensively in the laboratory over the last 20 years (see Salama 1999 and Tielens \& Peeters 2004 for reviews) owing to their astronomical significance as possible carriers of the unidentified infrared bands (UIRs, e.g. Allamandola et al. 1989). These studies have been performed almost exclusively in the uv, optical, and infrared regions of the electromagnetic spectrum. Very little is known, however, about the rotational spectra of small and polar PAHs, since microwave studies have been reported so far only for azulene $\left(\mathrm{C}_{10} \mathrm{H}_{8}\right.$, Huber et al. 2005 and references therein) and corannulene $\left(\mathrm{C}_{20} \mathrm{H}_{10}\right.$, Lovas et al. 2005).

In the present study, we have investigated the rotational spectra of selected small PAHs (see Fig. 1) employing Fourier transform microwave (FTM) spectroscopy (Balle \& Flygare 1981) using the spectrometer at Harvard (McCarthy et al. 1997, 2000) in combination with a heated nozzle recently developed for studies of low-volatility compounds
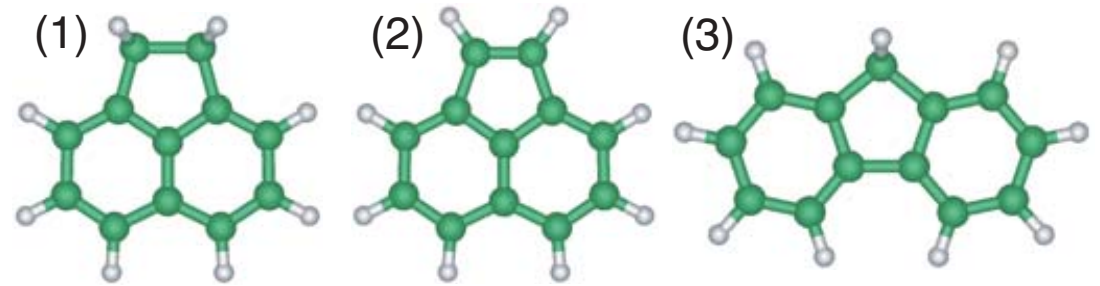

Figure 1. Molecular structures of acenaphthene $\left(\mathrm{C}_{12} \mathrm{H}_{10}, \mathbf{1}\right)$, acenaphthylene $\left(\mathrm{C}_{12} \mathrm{H}_{8}, \mathbf{2}\right)$ and fluorene $\left(\mathrm{C}_{13} \mathrm{H}_{10}, \mathbf{3}\right)$.

$\dagger$ Present address: Max-Planck-Institut für Radioastronomie, Auf dem Hügel 69, 53121 Bonn, Germany

$\ddagger$ Present address: Physique des interactions ioniques et moléculaires, Université de Provence, Centre de Saint Jérôme, 13397 Marseille Cedex 20, France 
(Thorwirth et al. 2005). Initial searches were guided by rotational constants obtained from quantum chemical calculations performed at the B3LYP/cc-pVTZ level of theory (see Table 1) using the program package Gaussian03 (Frisch et al. 2003). All three molecules exhibit $b$-type rotational spectra and are calculated to be moderately polar,

Table 1. Rotational constants (in $\mathrm{MHz}$ ) and dipole moments $\mu$ (in D) for (1), (2) and (3) as determined in the present study.

\begin{tabular}{lrrrr|rrr}
\hline \multirow{2}{*}{ Molecule } & \multicolumn{3}{c}{ B3LYP/cc-pVTZ } & \multicolumn{4}{c}{ Experiment } \\
& $A_{e}$ & $B_{e}$ & $C_{e}$ & $\mu$ & $A_{0}$ & $B_{0}$ & $C_{0}$ \\
\hline Acenaphthene (1) & 1416.5 & 1200.6 & 655.0 & 0.9 & 1410.3 & 1193.9 & 652.1 \\
Acenaphthylene (2) & 1520.7 & 1228.2 & 679.4 & 0.3 & 1511.8 & 1220.6 & 675.5 \\
Fluorene (3) & 2195.1 & 588.2 & 465.2 & 0.5 & 2176.2 & 586.7 & 463.6 \\
\hline
\end{tabular}

with dipole moments of order 0.3 to 0.9 D. Rotational transitions were found readily for all three molecules and based on improved predictions several tens of lines could be measured for each one. Rotational constants obtained from least-squares analyses of the experimental data are shown in Table 1 . As can be seen, the calculated equilibrium values and experimentally obtained ground state rotational constants agree very well, to within $1 \%$. Subsequently, selected rotational transitions of acenaphthene and fluorene could also be measured by standard millimeter wave absorption spectroscopy at $90 \mathrm{GHz}$.

The present investigation highlights the potential of FTM spectroscopy for the characterization of polar PAHs, including the nitrogen variants (PANHs; e.g., see Hudgins, this volume).

A detailed account of the present study will be given elsewhere.

\section{Acknowledgements}

This work was supported in part by NASA grant NAG5-9379 and NSF grant CHE0353693. S. Thorwirth is grateful to the Alexander von Humboldt-Foundation for a Feodor Lynen research fellowship. P. Theulé would like to thank the Swiss National Science Foundation for a research fellowship.

\section{References}

Allamandola, L.J., Tielens, A.G.G.M., \& Barker, J.R. 1989, Ap. J. Suppl. 71, 733

Balle, T.J. \& Flygare, W.H. 1981, Rev. Sci. Instrum. 51, 33

Frisch, M.J., Trucks, G. W., Schlegel, H.B. et al. 2003, Gaussian03, Revision B.04, Gaussian, Inc., Wallingford CT

Huber, S., Grassi, G., \& Bauder A. 2005, Mol. Phys. 103, 1395

Lovas, F.J., McMahon, R.J., Grabow, J.-U., Schnell, M., Mack, J., Scott, L.T., \& Kuczkowski, R. L. 2005, J. Am. Chem. Soc. 127, 4345

McCarthy, M.C., Travers, M.J., Kovács, A., Gottlieb, C.A., \& Thaddeus, P. 1997, Ap. J. Suppl. 113,105

McCarthy, M.C., Chen, W., Travers, M.J., \& Thaddeus, P. 2000, Ap. J. Suppl. 129, 611

Salama, F. 1999, in Solid Interstellar Matter: The ISO Revolution, eds. L. d'Hendecourt, C. Joblin, \& A. Jones (Springer-Verlag, New York), 65

Thorwirth, S., McCarthy, M.C., Gottlieb, C.A., Thaddeus, P., Gupta, H., \& Stanton, J.F. 2005, J. Chem. Phys. 123, 054326

Tielens, A.G.G.M. \& Peeters, E. 2004, in The Dense Interstellar Medium in Galaxies, eds. S. Pfalzner, C. Kramer, C. Staubmeier, \& A. Heithausen, Springer Proceedings in Physics, Vol. 91 (Springer-Verlag, Berlin), 497 